\title{
Influence of microheterogeneity on effective stress law for elastic properties of rocks
}

\author{
Radim Ciz ${ }^{1}$, Anthony F. Siggins ${ }^{2}$, Boris Gurevich ${ }^{3}$, and Jack Dvorkin ${ }^{4}$
}

\begin{abstract}
Understanding the effective stress coefficient for seismic velocity is important for geophysical applications such as overpressure prediction from seismic data as well as for hydrocarbon production and monitoring using time-lapse seismic measurements. This quantity is still not completely understood. Laboratory measurements show that the seismic velocities as a function of effective stress yield effective stress coefficients less than one and usually vary between 0.5 and 1. At the same time, theoretical analysis shows that for an idealized monomineral rock, the effective stress coefficient for elastic moduli (and therefore also for seismic velocities) will always equal one. We explore whether this deviation of the effective stress coefficient from unity can be caused by the spatial microheterogeneity of the rock. The results show that only a small amount (less than $1 \%$ ) of a very soft component is sufficient to cause this effect. Such soft material may be present in grain contact areas of many rocks and may explain the variation observed experimentally.
\end{abstract}

\section{INTRODUCTION}

Physical properties of porous rocks, such as seismic velocity, depend on both pore pressure $P_{p}$ and confining stress $\sigma_{c}$. The dependence of seismic velocity on pressure has been confirmed for a variety of rocks by laboratory measurements of elastic wave velocities in samples with varying pressure in pore fluids (Terzaghi, 1948; Wyllie et al., 1958; Todd and Simmons, 1972; Eberhart-Phillips et al., 1989; Zimmerman, 1991; Prasad and Manghnani, 1997; Siggins and Dewhurst, 2003). Because both confining stress and pore pressure vary in the subsurface, knowledge of acoustic velocities in rocks as functions of both confining stress and pore pressure is important for pre- dicting overpressure from seismic data (Dutta, 2002; Huffman, 2002; Sayers et al., 2002) and for correct interpretation of time-lapse and 4D seismic measurements (Tura and Lumley, 1999; Landrø, 2001; Vasco, 2004).

In general, for a rock subjected to a given confining stress $\sigma_{c}$, higher pore pressure $P_{p}$ corresponds to lower compressional and shear velocities. Confining stress has a similar effect (but with opposite sign) on seismic velocities. Usually, the dependence of seismic velocities on pore pressure and confining stress is described by an empirical relationship (Eberhart-Phillips et al., 1989; Zimmerman, 1991; Prasad and Manghnani, 1997):

$$
v_{p, s}\left(P_{d}\right)=a+k P_{d}-b \exp \left(-d P_{d}\right),
$$

where $P_{d}=P_{c}-P_{p}$ is the differential pressure and $P_{c}=-T r$ $\left(\sigma_{c}\right) / 3$ is the confining pressure. The coefficients $a, k, b$, and $d$ are empirical fitting parameters. Shapiro (2003) develops a model that relates the fitting parameters of equation 1 to physical properties of porous and fractured rocks. Further, experimental observations (Siggins and Dewhurst, 2003) show that equation 1 is stress-path dependent and hence not unique. The velocity-stress analysis can be simplified by introducing the concept of effective stress $\sigma_{e}$, in which the description of the stress dependence is given in terms of one single parameter, the effective stress.

Consider that some property of the rock, say $F$, depends only on the current stress state irrespective of the stress history and stress path. Thus, $F$ can be written as a function (some linear combination) of confining stress $\sigma_{c}$ and pore pressure $P_{p}$ :

$$
F \equiv F\left(\sigma_{c}, P_{p}\right)=F\left(\sigma_{e}\right),
$$

where the tensor $\sigma_{e}$

$$
\sigma_{e}=\sigma_{c}-n_{F} P_{p} \delta_{i j}
$$

is called effective stress and $n_{F}$ is called an effective stress coefficient for the property $F$. The property $F$ can be any measurable parameter

Manuscript received by the Editor 24 May 2007; revised manuscript received 29 August 2007; published online 12 December 2007.

${ }^{1}$ Freie Universität Berlin, Fachrichtung Geophysik, Berlin, Germany. E-mail: ciz@ geophysik.fu-berlin.de.

${ }^{2}$ CSIRO Petroleum, Melbourne, Australia. E-mail: tony.siggins@ csiro.au.

${ }^{3}$ Curtin University of Technology, Department of Exploration Geophysics, Perth, Western Australia, and CSIRO Petroleum, ARRC, Perth, Western Australia. E-mail: b.gurevich@curtin.edu.au.

${ }^{4}$ Stanford University, Geophysics Department, Stanford, California, U.S.A.E-mail: jack@ pangea.stanford.edu.

(C) 2008 Society of Exploration Geophysicists. All rights reserved. 
of the rock: density, overall volume (of a given rock sample), pore volume, ultrasonic compressional $v_{p}$, or shear $v_{s}$ velocity.

From the definition of the effective stress law, the total differential of equation 2 yields

$$
d F\left(\sigma_{c}, P_{p}\right)=\left[\frac{\partial F\left(\sigma_{c}, P_{p}\right)}{\partial \sigma_{c}}\right]_{P_{p}} d \sigma_{c}+\left[\frac{\partial F\left(\sigma_{c}, P_{p}\right)}{\partial P_{p}}\right]_{\sigma_{c}} d P_{p} .
$$

From equations 3 and 4, it follows that the effective stress coefficient for the arbitrary property $F$ can be defined as the ratio of the sensitivity of the property $F$ to changes in the pore pressure, to the sensitivity of the property $F$ to changes in the confining stress (Al-Wardy and Zimmerman, 2004):

$$
n_{F}=-\frac{\left(\frac{\partial F}{\partial P_{p}}\right)_{\sigma_{c}}}{\left(\frac{\partial F}{\partial \sigma_{c}}\right)_{P_{p}}}
$$

where the subscript outside the parentheses indicates a variable that is held constant. An in-depth analysis of the concept of effective stress in general has been performed by Robin (1973), Carroll and Katsube (1983), Zimmerman (1991), Berryman (1992), and Gurevich (2004). These studies show there is no universal effective stress coefficient for all rock properties, and different values apply for different physical quantities $F$.

The analysis of the elastic deformation of rocks shows that a porous rock made of a single linearly elastic and isotropic mineral has an effective stress coefficient for static drained bulk and shear moduli (and, approximately, for elastic wave velocities) equal to one (Gurevich, 2004). However, the results of many measurements (Todd and Simmons, 1972; Siggins and Dewhurst, 2003) consistently show values for the effective stress coefficient for seismic velocities smaller than one. Of course, real rocks never conform to the assumptions of the theoretical analysis: They are virtually never microhomogeneous (made of one mineral), and the minerals are almost always anisotropic. In addition, ultrasonic velocities are measured at high frequencies. However, it is not clear which of these effects causes the discrepancy between theoretical and numerical values of effective stress coefficients.

We analyze one of these effects, microheterogeneity. Using a simple spherical shell configuration, we investigate the degree of heterogeneity required to produce the measured values of effective stress coefficients. A similar approach using a cylindrical model is proposed by Al-Wardy and Zimmerman (2004).

\section{THEORETICAL BACKGROUND}

General effective stress rules, and a number of effective stress coefficients for various physical properties of inhomogeneous porous rocks, are derived by Berryman (1992) using the general stressstrain relationships given by Brown and Korringa (1975). Brown and Korringa (1975) define three independent constants for bulk moduli of the porous frame:

$$
\frac{1}{K^{*}}=\frac{1}{V}\left(\frac{\partial V}{\partial P_{d}}\right)_{P_{p}}
$$

$$
\begin{aligned}
& \frac{1}{K_{s}^{*}}=\frac{1}{V}\left(\frac{\partial V}{\partial P_{p}}\right)_{P_{d}}, \\
& \frac{1}{K_{\phi}^{*}}=\frac{1}{V_{\phi}}\left(\frac{\partial V_{\phi}}{\partial P_{p}}\right)_{P_{d}} .
\end{aligned}
$$

The fourth one, which is not independent, is given by

$$
\frac{1}{K_{P}^{*}}=\frac{1}{V_{\phi}}\left(\frac{\partial V_{\phi}}{\partial P_{d}}\right)_{P_{p}}
$$

where $V$ is total volume of the sample, $V_{\phi}=\phi V$ is pore volume, $\phi$ is porosity, and $P_{d}=P_{c}-P_{p}$ is differential pressure. The bulk modulus $K_{P}^{*}$ is related to $K^{*}$ and $K_{s}^{*}$ through the reciprocity theorem (Brown and Korringa, 1975; Berryman and Milton, 1991; Berryman, 1992):

$$
\frac{1}{K_{P}^{*}}=\frac{1}{\phi}\left(\frac{1}{K^{*}}-\frac{1}{K_{s}^{*}}\right)
$$

Brown and Korringa (1975) also derive an equation for the undrained bulk modulus $K_{\text {sat }}^{*}$. This equation is frequently called the generalized Gassmann's equation (Brown and Korringa, 1975; Berryman and Milton, 1991; Mavko et al., 1998) and reads

$$
K_{\mathrm{sat}}^{*}=K^{*}+\alpha^{* 2} M^{*}
$$

where

$$
\begin{aligned}
\frac{1}{M^{*}} & =\frac{\phi}{K_{f}}+\frac{\alpha^{*}}{K_{s}^{*}}-\frac{\phi}{K_{\phi}^{*}}, \\
\alpha^{*} & =1-\frac{K^{*}}{K_{s}^{*}} .
\end{aligned}
$$

In the case of the single mineral, the generalized Gassmann's equation 11 reduces to the standard Gassmann's equation (Gassmann, 1951; Berryman and Milton, 1991):

$$
K_{\mathrm{sat}}=K+\alpha^{2} M,
$$

where

$$
\begin{aligned}
\frac{1}{M} & =\frac{\phi}{K_{f}}+\frac{\alpha-\phi}{K_{s}}, \\
\alpha & =1-\frac{K}{K_{s}} .
\end{aligned}
$$

$K_{\text {sat }}$ and $K$ represent the undrained and drained bulk moduli, $K_{s}$ is the grain bulk modulus, and the two bulk moduli $K_{s}^{*}$ and $K_{\phi}^{*}$ from equation 12 are equal to the bulk modulus $K_{s}$ of the single granular constituent

$$
K_{s}^{*}=K_{\phi}^{*}=K_{s} .
$$

Brown and Korringa's equations 6-9 yield the isotropic-stress/volume-strain relationships for the total volume strain 


$$
\frac{d V}{V}=\frac{d P_{d}}{K^{*}}+\frac{d P_{p}}{K_{s}^{*}}
$$

and for pore volume strain

$$
\frac{d V_{\phi}}{V_{\phi}}=\frac{d P_{d}}{K_{p}^{*}}+\frac{d P_{p}}{K_{\phi}^{*}} .
$$

Based on these considerations, Berryman (1992) derives a number of effective stress coefficients for different properties of porous rocks. Following Berryman's work, we define here the effective stress coefficient for total volume and porosity. These definitions are used further to derive those coefficients for proposed single- and double-shell models and for comparison with the newly derived effective stress coefficient for drained bulk modulus.

The effective stress principle in equation 3 and the isotropicstress/volume-strain relationships in equation 18 define the effective stress coefficient $\alpha^{*}$ for the total volume:

$$
\frac{d V}{V}=\frac{d P_{d}}{K^{*}}+\frac{d P_{p}}{K_{s}^{*}}=\frac{1}{K^{*}}\left(d P_{c}-n_{V}^{*} d P_{p}\right),
$$

where the effective stress coefficient for total volume $n_{V}^{*} \equiv \alpha^{*}$ is given by equation 13 and for single-mineral porous rock is given by equation 16, respectively. In the following, we use the more conventional notation $\alpha^{*}$, or $\alpha$ in Gassmann's limit, respectively. The usual range of values for $\alpha^{*}$ is $\phi \leq \alpha^{*} \leq 1$.

The variation in porosity $\phi=V_{\phi} / V$ yields

$$
\frac{d V_{\phi}}{V_{\phi}}=\frac{d \phi}{\phi}+\frac{d V}{V} .
$$

Using equations 13,18 , and 19 , we obtain

$$
-\frac{d \phi}{\phi}=\left(\frac{\alpha-\phi}{\phi K^{*}}\right)\left(d P_{c}-n_{\phi}^{*} d P_{p}\right),
$$

where the effective stress coefficient for porosity $n_{\phi}^{*}$ is given by

$$
n_{\phi}^{*}=1-\frac{\frac{1}{K_{\phi}^{*}}-\frac{1}{K_{s}^{*}}}{\frac{1}{K_{P}^{*}}-\frac{1}{K^{*}}} .
$$

As noted by Berryman (1992), when only one solid constituent is present, relationship 17 yields the effective stress coefficient for porosity in Gassmann's limit $n_{\phi} \equiv 1$. The coefficient $n_{\phi}^{*}$ equals unity only when $K_{s}^{*}=K_{\phi}^{*}$ or when $K^{*}=0$. If the moduli satisfy $K_{s}^{*}>K_{\phi}^{*}$ $>0$, then $n_{\phi}^{*}<1$; however, if $K_{\phi}^{*}>K_{s}^{*}$ or if $K_{\phi}^{*}<0$, then $n_{\phi}^{*}>1$.

\section{EXPERIMENTAL OBSERVATIONS}

Stress-path-dependent velocity response in a selection of reservoir sandstones was investigated via ultrasonic velocity measurements on cores subjected to varied confining- and pore-pressure regimes. The sandstone samples were from three wells that intersect the Early Cretaceous Barrow Group in the Barrow Island subbasin in the Australian North West Shelf. They are from depths between 1700 and $2100 \mathrm{~m}$ and have undergone similar geologic histories since deposition. Porosities range from $6.6 \%$ to $24.1 \%$, and permeabilities vary from 0.01 to $1160 \mathrm{mD}$. The clay-content estimates vary from
$3 \%$ to $24 \%$ for individual samples. The sandstones were tested dry and oil saturated under ambient conditions and stepwise in a triaxial cell to a differential pressure of $60 \mathrm{MPa}$.

The stress paths simulated under experimental conditions the three mechanisms of overpressure (Siggins and Dewhurst, 2003):

1) Normal compaction-constant pore pressure (5 MPa) with increasing confining pressure reaching up to $65 \mathrm{MPa}$

2) Fluid expansion — constant confining pressure of $65 \mathrm{MPa}$ with pore pressure rising incrementally from 5 to $60 \mathrm{MPa}$

3) Disequilibrium compaction-constant differential pressure (10 MPa) with confining and pore pressures rising incrementally from 15 and $5 \mathrm{MPa}$, respectively, to 65 and $55 \mathrm{MPa}$, respectively

Ultrasonic measurements of $\mathrm{P}$ - and S-wave velocities were made along the length of the cores after pore-pressure equilibration at each pressure increment. Full waveforms were recorded for both transmitted $\mathrm{P}$ - and S-waves at nominal center frequencies of $800 \mathrm{kHz}(\mathrm{P}$ wave) and $400 \mathrm{kHz}$ (S-wave), respectively. The effective stress coefficient for compressional velocities $n_{v_{p}}$ are derived according to Todd and Simmons (1972) in Table 1. Seven different core samples show the deviation of the effective stress coefficient from unity. To explain this observation by means of the presence of microheterogeneity, we analyze this further in the double-shell model. The details on the experimental data can be found elsewhere (Siggins and Dewhurst, 2003; Ciz et al., 2005; Dodds et al., 2007). Figures similar to those of Todd and Simmons (1972) are shown in these papers.

\section{ANALYTICAL SOLUTIONS}

As discussed, one possible factor that may cause observed values of effective stress coefficient for seismic velocities $n_{v}$ to be smaller than the theoretical value $n_{v_{p}}=1$ is microheterogeneity. To analyze this effect, we compute effective stress coefficients for a simple spherical shell configuration, where a spherical pore is surrounded by spherical shells of two linear and isotropic minerals (a doubleshell model, Figure 1a). However, before analyzing the double-shell model, we first consider the reference case of a single-shell model (Figure 1b).

Table 1. Experimentally derived effective stress coefficients $\boldsymbol{n}_{\boldsymbol{v}}$ for ultrasonic velocities $\boldsymbol{v}_{p}$ for eight different oil-saturated sandstone samples. The confining pressure varies from 10 to $65 \mathrm{MPa}$, and the pore pressure varies from 5 to $60 \mathrm{MPa}$ in three different stress paths simulated in the experiments.

\begin{tabular}{lccc}
\hline & & & $\begin{array}{c}\text { Effective stress coefficient } \\
\text { for P-wave velocity } \\
n_{v_{p}}\end{array}$ \\
\hline 1207 & 20.4 & 10 & 0.58 \\
1203 & 20.6 & 24 & 0.79 \\
1204 & 18.7 & 6.3 & 1.00 \\
1206 & 19.9 & 4 & 0.60 \\
$05 \mathrm{~V}$ & 23.7 & 3 & 0.76 \\
$08 \mathrm{~V}$ & 20.3 & 3 & 0.68 \\
$15 \mathrm{~V}$ & 24.1 & 4 & 0.77 \\
$12 \mathrm{~V}$ & 6.6 & 4 & 0.35 \\
\hline
\end{tabular}




\section{Single-shell model}

Figure $1 \mathrm{~b}$ shows an idealized rock represented by a spherical shell with inner radius $R_{p}$ and outer radius $R_{c}$. The shell itself is made of a solid with the bulk modulus $K_{s}$, shear modulus $\mu_{s}$, and density $\rho_{s}$. We assume that within the range of stress fields considered, the solid grain material is linearly elastic, that is, $K_{s}$ and $\mu_{s}$ are constants. The inner sphere represents the pore filled with a fluid with the bulk modulus $K_{f}$, viscosity $\eta$, and density $\rho_{f}$.

Suppose that the shell is in the static equilibrium state when it is subjected to the confining stress $P_{c}$ on the outer sphere and pore pressure $P_{p}$ on the inner sphere. Let $\sigma$ be the stress field within the solid. To obtain the effective stress coefficient for drained bulk modulus for our model, we must solve the elasticity equations for static equilibrium. The equations of equilibrium for an inhomogeneous elastic medium are, in general, three coupled partial differential equations with three unknown components of the displacement vector (Landau and Lifshitz, 1959; Pilant, 1979).

However, for the model with spherical symmetry, where the radial displacement $u_{r}$ is the only nonzero component of displacement, the problem simplifies considerably. Two of the three equations of stress equilibrium are satisfied identically. The third one and Hooke's law provide the solution for the exact moduli for the single-shell model (for details of the derivation, see Appendix A):

$$
\begin{aligned}
\frac{1}{K} & =\frac{3}{1-\phi}\left(\frac{1}{3 K_{s}}+\frac{\phi}{4 \mu_{s}}\right), \\
\frac{1}{K_{P}} & =\frac{3}{1-\phi}\left(\frac{1}{3 K_{s}}+\frac{1}{4 \mu_{s}}\right),
\end{aligned}
$$

and

$$
K_{\phi}=K_{s}
$$

Further, these moduli (equations 24-26) enable us to derive the effective stress coefficients for total volume $\alpha$ by substituting them into equation 16 . The effective stress coefficient $\alpha$ for the singleshell model then reads

$$
\alpha=1-\frac{K}{K_{s}}=\frac{\phi\left(3 K_{s}+4 \mu_{s}\right)}{\left(3 \phi K_{s}+4 \mu_{s}\right)} .
$$

Substituting equations 24-26 into equation 23 for the effective stress coefficient for porosity and into general equation 5 , we obtain the effective stress coefficients for porosity $n_{\phi}$ and drained bulk modulus $n_{K}$ for the single-shell model to be unity:
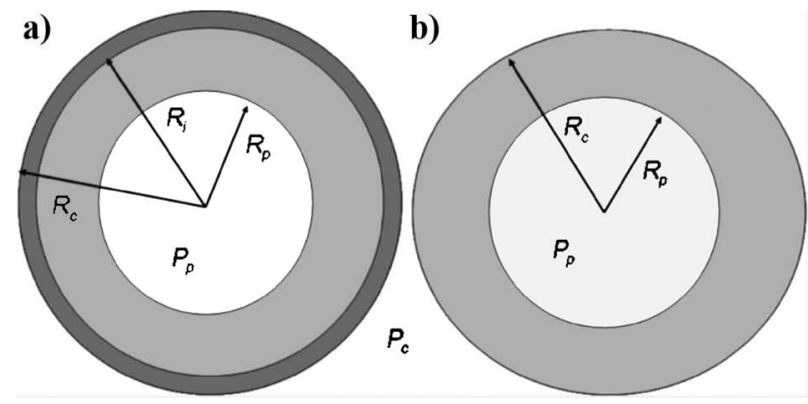

Figure 1. (a) Double-shell and (b) single-shell models. Pore pressure $P_{p}$ is applied from inside and confining pressure $P_{c}$ from outside the spherical shell.

$$
n_{\phi}=n_{K}=1 \text {. }
$$

This agrees with Gassmann's limit (Berryman and Milton, 1991; Berryman, 1992; Gurevich, 2004) when only a single mineral is present.

\section{Double-shell model}

The effect of microheterogeneity can be modeled schematically using a double-shell model shown in Figure 1a. Elastic moduli of the inner and outer shell are denoted by superscripts 1 and 2, respectively. We follow the same approach as for the single-shell model. Hence, to obtain the effective stress coefficients for the double-shell model, we need to solve the elasticity equations for a pore embedded into the elastic solid composed of two elastic shells. The spherical shell has inner radius $R_{p}$, outer radius $R_{c}$, and radius of the spherical boundary between these two shells $R_{i}$. The inner and outer solid components are assumed to be linear elastic materials described by a constant bulk and shear moduli $K_{s}^{(1)}, \mu_{s}^{(1)}$ for the inner shell material and $K_{s}^{(2)}, \mu_{s}^{(2)}$ for the outer shell solid.

We assume that the shell is in a static equilibrium state when it is subjected to the confining pressure $P_{c}$ on the outer sphere and pore pressure $P_{p}$ on the inner sphere. These acting pressures cause changes in the stress field $\sigma$, strain $e$, and displacement $u$, which can be described by the static equilibrium equation A-1 or A-5, respectively, and the stress/strain relationship in each shell as given by Hooke's law (equation A-2). The details of the derivation for the double-shell model are given in Appendix B.

The resulting cumbersome equation B-10 for drained bulk modulus $K$ can be simplified using the following substitutions:

$$
R_{i}=R_{c}(1-\varepsilon) K_{s}^{(2)}=a \varepsilon K_{s}^{(1)} \mu_{s}^{(2)}=b \varepsilon K_{s}^{(1)} .
$$

In the limit $\varepsilon \rightarrow 0$, the drained bulk modulus yields

$K=\frac{4 K_{s}^{(1)} \mu_{s}^{(1)}\left(R_{p}-R_{c}\right)\left(R_{p}^{2}+R_{p} R_{c}+R_{c}^{2}\right)(4 b+3 a)}{\left(12 \mu_{s}^{(1)} a+16 \mu_{s}^{(1)} b+36 \mu_{s}^{(1)}\right) R_{c}^{3}+\left(9 K_{s}^{(1)} a-36 \mu_{s}^{(1)}+12 K_{s}^{(1)} b\right) R_{p}^{3}}$,

where $a$ and $b$ are parameters given by substitutions in expressions 29. The results are discussed in more detail in the next section.

\section{RESULTS AND DISCUSSION}

We have shown that the single-shell model, representing the rock composed from homogeneous material, yields an effective stress coefficient for porosity and drained bulk modulus equal to unity. The Biot's effective stress coefficient $\alpha$ for total volume is given by equation 27 and depends on drained and grain bulk moduli $K$ and $K_{s}$.

Figure 2a shows the comparison between fluid-saturated bulk moduli computed for the double-shell model by the Brown and Korringa equation and Gassmann's equation. The Gassmann fluid substitution is computed with the effective grain bulk modulus obtained from the average of Hashin-Shtrikman bounds (Hashin and Shtrikman, 1963). The results show that Gassmann's equation is consistent with Brown and Korringa's equation for the wide range of realistic parameters of rocks. The inconsistency is shown for unrealistic parameters (very high contrast in elastic moduli) by Berge and Berryman (1995). Figure $2 b$ shows the difference between the undrained and drained bulk moduli in dependence of porosity. The difference increases from zero to the value of the bulk modulus of pore-filling 
fluid $K_{f}=2.2 \mathrm{GPa}$ for porosity $\phi=1$. The parameters of the model are the bulk and shear moduli of the inner spherical shell are $K_{s}^{(1)}$ $=37 \mathrm{GPa}$ and $\mu_{s}^{(1)}=43 \mathrm{GPa}$ and the outer shell material has bulk and shear moduli $K_{s}^{(1)}=3.7 \mathrm{GPa}$ and $\mu_{s}^{(1)}=4.4 \mathrm{GPa}$, representing a soft microlayer of heterogeneity with thickness $h=R_{c}-R_{p}$ $=1 e-2$.

The effective stress coefficients are shown in Figure 3. The effective stress coefficients for total volume in single- and double-shell models are the same because of the small volume fraction of the soft component. However, even a very tiny amount of the soft component causes the variation of effective stress coefficients from unity for porosity and drained bulk moduli in the double-shell model. The effective stress coefficient for porosity $n_{\phi}^{*}$ in the double-shell model is bigger than one. This is in agreement with theoretical analysis of Berryman (1992) and Berge and Berryman (1995): The effective stress coefficient for drained bulk modulus $n_{K}^{*}$ is less than one. Thus, the presence of microheterogeneity can be the cause of variation of effective stress coefficient from unity observed experimentally (Siggins and Dewhurst, 2003). This is consistent with a thin clay coating on the sandstone grains and grain contacts.

The experimentally obtained effective stress coefficients are derived for seismic velocities, whereas we have derived the effective stress coefficient for drained bulk modulus. However, we assume that in the stress range considered, the effect of stress on both fluid properties and overall rock density is small; hence, these two effective stress coefficients (for seismic velocities and for drained bulk modulus) show similar behavior. Therefore, our spherical model represents the idealized situation for analyzing of the effect of microheterogeneity on the effective stress coefficients.

\section{a)}

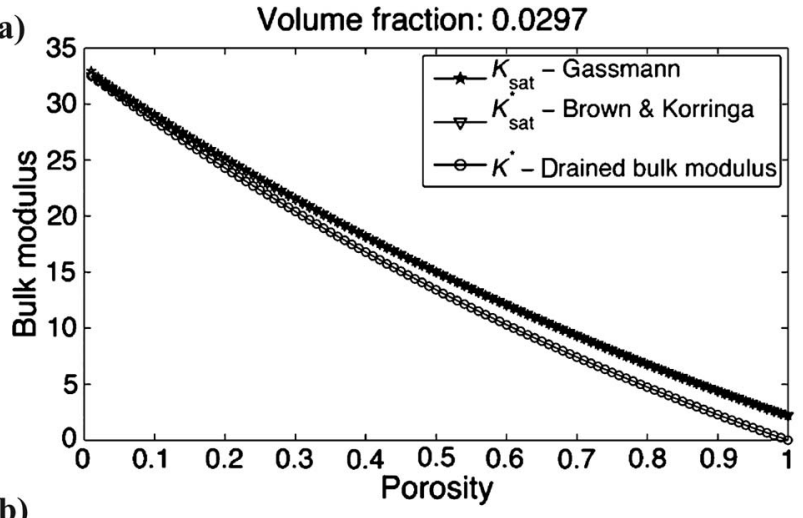

b)

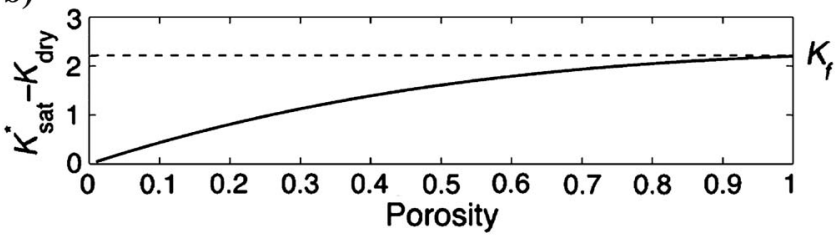

Figure 2. (a) Saturated and drained bulk moduli derived for the double-shell model in dependence on porosity. (b) Difference between undrained and drained bulk moduli from (a) in dependence on porosity. The bulk and shear moduli of the inner spherical shell are $K_{s}^{(1)}$ $=37 \mathrm{GPa}$ and $\mu_{\mathrm{s}}^{(1)}=43 \mathrm{GPa}$. The outer-shell material has bulk and shear moduli $K_{s}^{(2)}=3.7 \mathrm{GPa}$ and $\mu_{s}^{(2)}=4.4 \mathrm{GPa}$, representing a soft microlayer of heterogeneity with the thickness $h=R_{c}-R_{p}$ $=1 e-2$ and bulk modulus of pore fluid $K_{f}=2.2 \mathrm{GPa}$.
Figure 4 shows the variation of effective stress coefficient from unity for a negligible amount of very soft component of the outer shell. In this case, the outer-shell material has the bulk and shear moduli $K_{s}^{(1)}=0.037 \mathrm{GPa}$ and $\mu_{s}^{(1)}=0.043 \mathrm{GPa}$, representing a soft microlayer of heterogeneity with the thickness $h=R_{c}-R_{p}=1 e$ -6 . The outer-shell material having such parameters can also represent a very small amount of gas bounded on the grains. The small amount of gas significantly decreases in seismic velocities.

At this stage, a question must be asked: Is this scenario (with a huge contrast) plausible? We do not know. Coating might result from trapped gas or some unknown alteration. We want also to underline the usefulness of the negative result of the paper: If heterogeneity is responsible for this, the contrast must be huge. But this is possible with a tiny amount of coating so small, it does not affect the effective modulus.

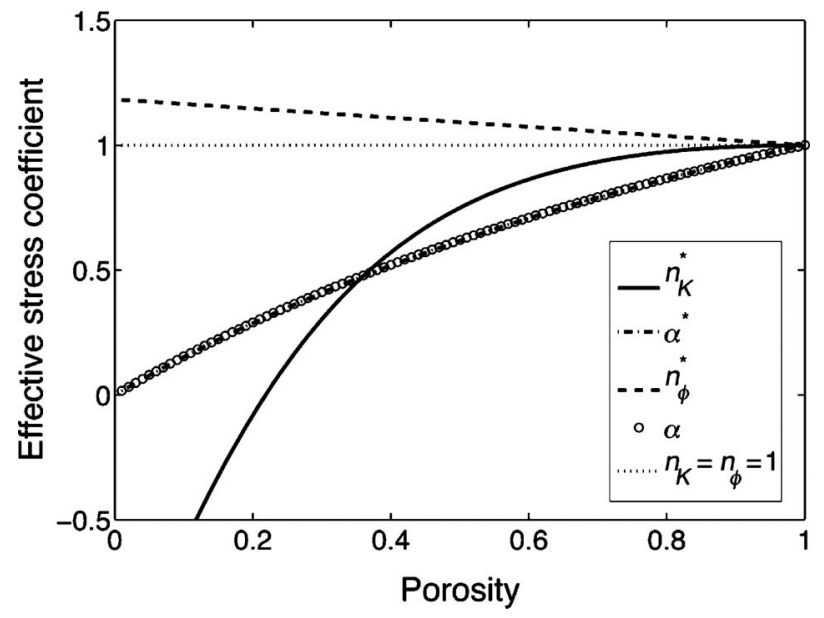

Figure 3. The effective stress coefficient for total volume $\alpha$ and for the drained bulk modulus $n_{k}$ versus porosity as derived for the double-shell model. The model parameters are the same as in Figure 2.

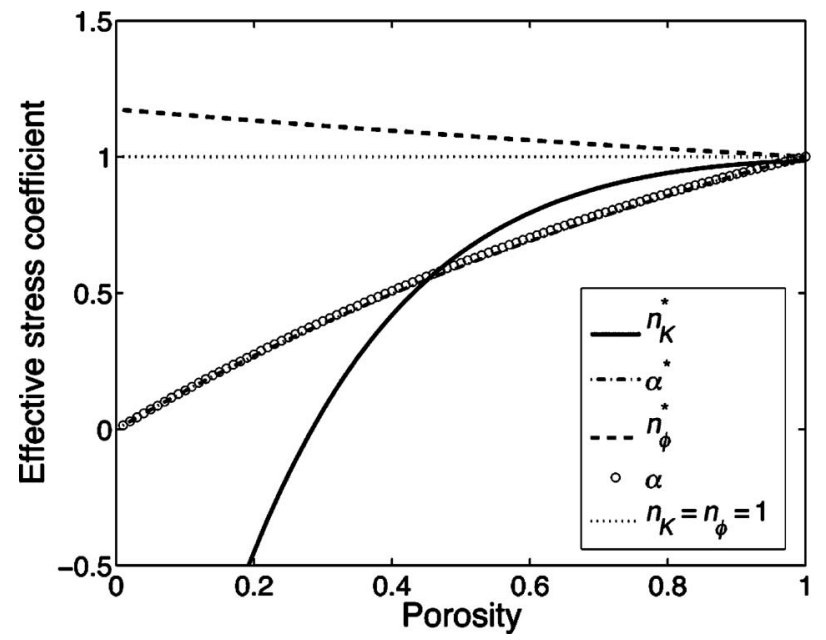

Figure 4. The effective stress coefficient for total volume $\alpha$ and for the drained bulk modulus $n_{k}$ versus porosity as derived for the double-shell model. The model parameters are the bulk and shear moduli $K_{s}^{(1)}=0.037 \mathrm{GPa}$ and $\mu_{s}^{(1)}=0.043 \mathrm{GPa}$ representing a soft microlayer of heterogeneity with a thickness $h=R_{c}-R_{p}=1 e-6$. 


\section{CONCLUSIONS}

We have analyzed the effect of spatial microinhomogeneity on the effective stress coefficient for elastic moduli of porous rocks using a double-shell model: a system of two concentric spherical shells made of two distinct elastic solid materials surrounding a spherical pore. This analysis shows that spatial microinhomogeneity can indeed significantly affect the effective stress coefficient and cause its deviation from unity, but only if the contrast between the two materials is very large - more than one order of magnitude. In particular, this can occur if a small amount of very soft component is added to the main grain material. Although, to have a noticeable effect on the effective stress coefficient the elastic moduli of the soft component must be 10-30 times smaller than the moduli of the main grain material, its volume concentration can be very small, such that it has negligible effect on the effective moduli of the overall material.

It is not clear whether this scenario is realistic and if the spatial microinhomogeneity is indeed the cause of the deviation of the experimentally observed effective stress coefficients from unity. The very small amount of the soft component sufficient to produce such an effect suggests that we may not be able to notice the presence of such microinhomogeneity from acoustic or other petrophysical measurements.

\section{ACKNOWLEDGMENTS}

The work was supported by the CSIRO Postdoctoral Fellowship Program, the PHASE consortium at the Freie Universität Berlin, and the Pre-Drill Pore Pressure Prediction Program of the Australian Petroleum Centre for Cooperative Research Centre (APCRC). The authors thank David Dewhurst, Kevin Dodds, and Serge Shapiro for useful advice and discussions.

\section{APPENDIX A \\ DERIVATION OF SOLUTION FOR THE SINGLE-SHELL MODEL} reads

The equation for the stress equilibrium in spherical coordinates

$$
\frac{\partial \sigma_{r r}}{\partial r}+\frac{2\left(\sigma_{r r}-\sigma_{\theta \theta}\right)}{r}=0
$$

where the elastic stress-strain relationship is given by Hooke's law:

$$
\begin{aligned}
\sigma_{r r}=K_{s} v+2 \mu_{s}\left(e_{r r}-\frac{1}{3} v\right) \\
\sigma_{\theta \theta}=K_{s} v+2 \mu_{s}\left(e_{\theta \theta}-\frac{1}{3} v\right),
\end{aligned}
$$

assuming only a linearized expression for elastic strain

$$
e_{r r}=\frac{\partial u_{r}}{\partial r}, \quad e_{\theta \theta}=\frac{u_{r}}{r}, \quad v=\frac{\partial u_{r}}{\partial r}+\frac{2 u_{r}}{r} .
$$

Substituting equations A-2 and A-3 into equation A-1 yields the equation of equilibrium for isotropic bodies in terms of displacements (Landau and Lifshitz, 1959; Pillant, 1979):

$$
\left(K_{s}+\frac{4}{3} \mu_{s}\right) \nabla^{2} u_{r}=\left(K_{s}+\frac{4}{3} \mu_{s}\right)\left(\frac{\partial^{2}}{\partial r^{2}}+\frac{2}{r} \frac{\partial}{\partial r}\right) u_{r}=0 .
$$

Thus, equation A-4 becomes $\nabla\left(\nabla . u_{r}\right)=0$ and in spherical coordinates reads

$$
\nabla \cdot u_{r}=\frac{1}{r^{2}} \frac{\partial}{\partial r}\left(r^{2} u_{r}\right)=\text { constant }=A .
$$

The general solution to equation A-5 yields (Landau and Lifshitz, 1959)

$$
u_{r}=\frac{A r}{3}+\frac{B}{r^{2}}
$$

Integration constants $A$ and $B$ are obtained by solving boundary conditions on the inner and outer surface of the spherical shell:

$$
\sigma_{r r}\left(r \equiv R_{p}\right)=P_{p}
$$

and

$$
\sigma_{r r}\left(r \equiv R_{c}\right)=P_{c}
$$

The solution yields integration constants $A$ and $B$ :

$$
\begin{aligned}
& A=\frac{1}{K_{s}} \frac{R_{p}^{3} P_{p}-R_{c}^{3} P_{c}}{R_{c}^{3}-R_{p}^{3}}, \\
& B=\frac{1}{4 \mu_{s}} \frac{R_{p}^{3} R_{c}^{3}\left(P_{p}-P_{c}\right)}{R_{c}^{3}-R_{p}^{3}} .
\end{aligned}
$$

Substituting the integration constants $A$ and $B$ (equations A-9 and A-10) into the general solution in equation A-6 and introducing the porosity of our simple spherical model $\phi=\left(R_{p} / R_{c}\right)^{3}$ gives the radial displacement $u_{r}$ as a function of applied pressures on the inner and outer shell surface:

$u_{r}=\frac{r}{3 K_{s}(1-\phi)}\left(\phi P_{p}-P_{c}\right)+\frac{R_{p}^{3}}{4 r^{2} \mu_{s}(1-\phi)}\left(P_{p}-P_{c}\right)$.

Equation A-11 represents the solution for the solid displacement of the single spherical shell loaded by the pore pressure $P_{p}$ on the inner surface and by the confining pressure $P_{c}$ on the outer surface. The displacement changes on the inner and outer shell surfaces because $d P_{p}$ and $d P_{c}$ are

$$
\begin{aligned}
d u_{r}(r \equiv & \left.R_{p}\right)=\frac{R_{p}}{1-\phi}\left[\left(\frac{\phi}{3 K_{s}}+\frac{1}{4 \mu_{s}}\right) d P_{p}\right. \\
& \left.-\left(\frac{1}{3 K_{s}}+\frac{1}{4 \mu_{s}}\right) d P_{c}\right]
\end{aligned}
$$


and

$$
\begin{aligned}
d u_{r}(r \equiv & \left.R_{c}\right)=\frac{R_{c}}{1-\phi}\left[\phi\left(\frac{1}{3 K_{s}}+\frac{1}{4 \mu_{s}}\right) d P_{p}\right. \\
& \left.-\left(\frac{1}{3 K_{s}}+\frac{\phi}{4 \mu_{s}}\right) d P_{c}\right] .
\end{aligned}
$$

Corresponding changes in the pore volume $V_{\phi}=4 \pi R_{p}^{3} / 3$ and total volume $V=4 \pi R_{c}^{3} / 3$ are

$$
d V_{\phi}=\frac{3 V_{\phi}}{1-\phi}\left[\left(\frac{\phi}{3 K_{s}}+\frac{1}{4 \mu_{s}}\right) d P_{p}-\left(\frac{1}{3 K_{s}}+\frac{1}{4 \mu_{s}}\right) d P_{c}\right]
$$

and

$d V=\frac{3 V}{1-\phi}\left[\phi\left(\frac{1}{3 K_{s}}+\frac{1}{4 \mu_{s}}\right) d P_{p}-\left(\frac{1}{3 K_{s}}+\frac{\phi}{4 \mu_{s}}\right) d P_{c}\right]$

Using equations A-14 and A-15 and Brown and Korringa's isotropic-stress/volume-strain relationships 18 and 19 yields the exact moduli for the single-shell model (equations 24-26).

\section{APPENDIX B}

\section{DERIVATION OF SOLUTION FOR DOUBLE-SHELL MODEL}

We assume the shell is in a static equilibrium state when it is subjected to the confining pressure $P_{c}$ on the outer sphere and pore pressure $P_{p}$ on the inner sphere. These acting pressures cause changes in the stress field $\sigma$, strain $e$, and displacement $u$, which can be described by the static equilibrium equation A-1 or A-5, respectively; the stress/strain relationship in each shell is given by Hooke's law A-2. The general solutions for inner and outer shell layers in spherical coordinates according to equation A-6 reads

$$
u_{r}^{(q)}=\frac{A_{q} r}{3}+\frac{B_{q}}{r^{2}},
$$

where $q=1,2$ represent the solid shell components 1 and 2, respectively. Integration constants $A_{q}$ and $B_{q}$ are obtained by solving the following boundary conditions. Radial stress component $\sigma_{r r}^{(1)}$ equals pore pressure $P_{p}$ on the inner shell boundary:

$$
\sigma_{r r}^{(1)}\left(r \equiv R_{p}\right)=P_{p}
$$

Radial stress component $\sigma_{r r}^{(2)}$ equals confining pressure $P_{c}$ on the outer shell:

$$
\sigma_{r r}^{(2)}\left(r \equiv R_{c}\right)=P_{c}
$$

Continuity of stress on the inner shell interface $r=R_{i}$ :

$$
\sigma_{r r}^{(1)}\left(r \equiv R_{i}\right)=\sigma_{r r}^{(2)}\left(r \equiv R_{i}\right) .
$$

Continuity of the displacement on the inner shell interface $r=R_{i}$ :

$$
u_{r}^{(1)}\left(r \equiv R_{i}\right)=u_{r}^{(2)}\left(r \equiv R_{i}\right)
$$

Solving these $4 \times 4$ equations, we obtain the unknown constants $A_{1}$, $A_{2}, B_{1}$, and $B_{2}$ :

$$
\begin{aligned}
& A_{1}= \frac{1}{\Delta}\left\{\left[\left(4 \mu_{s}^{(1)} \mu_{s}^{(2)}+3 K_{s}^{(2)} \mu_{s}^{(2)}\right) R_{i}^{3}\right.\right. \\
&\left.+\left(3 K_{s}^{(2)} \mu_{s}^{(1)}-3 K_{s}^{(2)} \mu_{s}^{(2)}\right) R_{c}^{3}\right] R_{p}^{3} P_{p} \\
&\left.-\left(3 K_{s}^{(2)} \mu_{s}^{(1)}+4 \mu_{s}^{(1)} \mu_{s}^{(2)}\right) R_{c}^{3} R_{i}^{3} P_{c}\right\}, \\
& B_{1}=\frac{1}{\Delta}\left\{\left[\left(\frac{3}{4} K_{s}^{(1)} K_{s}^{(2)}+K_{s}^{(2)} \mu_{s}^{(2)}\right) R_{c}^{3}\right.\right. \\
&\left.+\left(K_{s}^{(1)} \mu_{s}^{(2)}+K_{s}^{(2)} \mu_{s}^{(2)}\right) R_{i}^{3}\right] R_{p}^{3} R_{i}^{3} P_{p} \\
&\left.-\left(\frac{3}{4} K_{s}^{(1)} K_{s}^{(2)}+K_{s}^{(1)} \mu_{s}^{(2)}\right) R_{c}^{3} R_{i}^{3} R_{p}^{3} P_{c}\right\},
\end{aligned}
$$

$$
A_{2}=\frac{1}{\Delta}\left\{\left[\left(3 K_{s}^{(1)} \mu_{s}^{(1)}-3 K_{s}^{(1)} \mu_{s}^{(2)}\right) R_{p}^{3}\right.\right.
$$

$$
\left.-\left(3 K_{s}^{(1)} \mu_{s}^{(2)}+4 \mu_{s}^{(1)} \mu_{s}^{(2)}\right) R_{i}^{3}\right] R_{c}^{3} P_{c}
$$$$
\left.+\left(3 K_{s}^{(1)} \mu_{s}^{(2)}-4 \mu_{s}^{(1)} \mu_{s}^{(2)}\right) R_{i}^{3} R_{p}^{3} P_{p}\right\} \text {, }
$$

$$
\begin{aligned}
B_{2}= & \frac{1}{\Delta}\left\{\left[\left(K_{s}^{(1)} \mu_{s}^{(1)}-K_{s}^{(2)} \mu_{s}^{(1)}\right) R_{i}^{3}\right.\right. \\
& \left.-\left(\frac{3}{4} K_{s}^{(1)} K_{s}^{(2)}+K_{s}^{(2)} \mu_{s}^{(1)}\right) R_{p}^{3}\right] R_{c}^{3} R_{i}^{3} P_{c} \\
& \left.+\left(\frac{3}{4} K_{s}^{(1)} K_{s}^{(2)}+K_{s}^{(1)} \mu_{s}^{(1)}\right) R_{c}^{3} R_{i}^{3} R_{p}^{3} P_{p}\right\},
\end{aligned}
$$

$$
\begin{aligned}
\Delta= & \mu_{s}^{(1)} K_{s}^{(2)}\left(3 K_{s}^{(1)}+4 \mu_{s}^{(2)}\right) R_{i}^{3} R_{c}^{3}-K_{s}^{(1)} \mu_{s}^{(2)} \\
& \times\left(4 \mu_{s}^{(1)}+3 K_{s}^{(2)}\right) R_{i}^{3} R_{p}^{3}+4 \mu_{s}^{(1)} \mu_{s}^{(2)}\left(K_{s}^{(1)}-K_{s}^{(2)}\right) R_{i}^{6} \\
& +3 K_{s}^{(1)} K_{s}^{(2)}\left(\mu_{s}^{(2)}-\mu_{s}^{(1)}\right) R_{c}^{3} R_{p}^{3} .
\end{aligned}
$$

Substituting into the general solution in equation B-1 yields the particular solution of the elasticity problem which defines local displacement in response to the changes in pore and confining pressure. This solution enables derivation of bulk moduli given by Brown and Korringa's equations 18 and 19 for our double-shell model:

$$
\begin{aligned}
\frac{1}{K^{*}}= & \frac{3}{4} \frac{1}{\Delta_{1}}\left\{\phi R _ { i } ^ { 3 } \left[4 \mu_{s}^{(1)}\left(K_{s}^{(2)}-K_{s}^{(1)}\right) R_{i}^{3}\right.\right. \\
& \left.-K_{s}^{(1)}\left(4 \mu_{s}^{(1)}+3 K_{s}^{(2)}\right) R_{p}^{3}-4 \mu_{s}^{(1)}\left(K_{s}^{(1)}+\mu_{s}^{(2)}\right) R_{c}^{3}\right] \\
& \left.-4 K_{s}^{(1)}\left(\mu_{s}^{(2)}-\mu_{s}^{(1)}\right) \phi R_{p}^{3} R_{c}^{3}\right\},
\end{aligned}
$$




$$
\begin{aligned}
\Delta_{1}= & 3 K_{s}^{(1)} K_{s}^{(1)}\left(\mu_{s}^{(1)}-\mu_{s}^{(2)}\right) R_{p}^{6}-K_{s}^{(2)} \mu_{s}^{(1)}\left(3 \mu_{s}^{(1)}-4 \mu_{s}^{(2)}\right) \\
& \times R_{p}^{3} R_{i}^{3}-\mu_{s}^{(1)}\left(3 K_{s}^{(1)}-4 \mu_{s}^{(2)}\right) \phi R_{c}^{3} R_{i}^{3}-3 K_{s}^{(1)}\left(\mu_{s}^{(2)}\right. \\
& \left.-\mu_{s}^{(1)}\right) \phi R_{p}^{3} R_{c}^{3},
\end{aligned}
$$

$$
\begin{aligned}
\frac{1}{K_{\phi}^{*}}= & \frac{1}{\Delta_{2}}\left\{3 \mu_{s}^{(2)}\left(K_{s}^{(1)}-K_{s}^{(2)}\right)\right. \\
& \times \phi R_{i}^{6}+3 K_{s}^{(2)}\left(\mu_{s}^{(1)}-\mu_{s}^{(2)}\right) \phi R_{p}^{6}+\mu_{s}^{(2)}\left(3 K_{s}^{(1)}\right. \\
& \left.+4 \mu_{s}^{(1)}\right) \phi R_{i}^{3} R_{p}^{3}+\left(3 K_{s}^{(2)} \mu_{s}^{(2)}-3 \mu_{s}^{(2)} K_{s}^{(1)}\right. \\
& \left.\left.-3 \mu_{s}^{(1)} K_{s}^{(2)}-4 \mu_{s}^{(1)} \mu_{s}^{(2)}\right) R_{i}^{3} R_{p}^{3}\right\},
\end{aligned}
$$$$
\frac{1}{K_{s}^{*}}=\frac{1}{\Delta_{2}}\left\{3 \mu_{s}^{(1)}\left(K_{s}^{(1)}-K_{s}^{(2)}\right) \phi R_{i}^{6}+3 \mu_{s}^{(1)}\left(K_{s}^{(2)}-K_{s}^{(1)}\right)\right.
$$$$
\times \phi R_{p}^{3} R_{i}^{3}+\mu_{s}^{(2)}\left(4 \mu_{s}^{(1)}+3 K_{s}^{(1)}\right) \phi^{2} R_{c}^{3} R_{i}^{3}-\mu_{s}^{(1)}
$$$$
\times\left(4 \mu_{s}^{(2)}+3 K_{s}^{(1)}\right) \phi R_{i}^{3} R_{c}^{3}+3 K_{s}^{(1)}\left(\mu_{s}^{(1)}\right.
$$$$
\left.\left.-\mu_{s}^{(2)}\right) \phi R_{p}^{3} R_{c}^{3}\right\}
$$

$$
\begin{aligned}
\Delta_{2}= & 3 K_{s}^{(1)} K_{s}^{(2)}\left(\mu_{s}^{(1)}-\mu_{s}^{(2)}\right) R_{i}^{6}-K_{s}^{(2)} \mu_{s}^{(1)} \\
& \times\left(K_{s}^{(1)}-\mu_{s}^{(2)}\right) R_{p}^{3} R_{i}^{3}+4 \mu_{s}^{(1)} \mu_{s}^{(2)}\left(K_{s}^{(2)}-K_{s}^{(1)}\right) \\
& \times \phi R_{i}^{6}+K_{s}^{(1)} \mu_{s}^{(2)}\left(4 \mu_{s}^{(1)}+3 K_{s}^{(2)}\right) \phi R_{p}^{3} R_{i}^{3} .
\end{aligned}
$$

By substituting these moduli into equations 13 and 23, we get effective stress coefficients for total volume and porosity for the double-shell model. To obtain the effective stress coefficient for the drained bulk modulus $n_{K}$, we express the displacement changes $d u_{i}$ on the inner and outer shell boundary and on the inner shell interface caused by the pore- and confining-pressure changes $d P_{p}$ and $d P_{c}$ using equation B-1:

$$
d u_{r}=w_{r} d P c+v_{r} d P_{p}
$$

where $r=\left\{R_{p}, R_{i}, R_{c}\right\}$. Using the general definition of the effective stress coefficient 5 and equation B-10 yields the effective stress coefficient for drained bulk modulus $n_{K}$ :

$$
n_{K}=\frac{\sum_{r} \frac{\partial K^{*}}{\partial r} w_{r}}{\sum_{r} \frac{\partial K^{*}}{\partial r} v_{r}} .
$$

The explicit expression is cumbersome and thus we show the results in graphical form (Figures 2-4).

\section{REFERENCES}

Al-Wardy, W., and R. W. Zimmerman, 2004, Effective stress law for the permeability of clay-rich sandstones: Journal of Geophysical Research, 109, B04203.

Berge, P. A., and J. G. Berryman, 1995, Realizability of negative pore compressibility in poroelastic composites: Journal of Applied Mechanics, 62, 1053-1061.

Berryman, J. G., 1992, Effective stress for transport properties of inhomogeneous porous rock: Journal of Geophysical Research, 97, 17409-17424.

Berryman, J. G., and G. W. Milton, 1991, Exact results for generalized Gassmanns equations in composite porous media with two constituents: Geophysics, 56, 1950-1960.

Brown, R. J. S., and J. Korringa, 1975, On the dependence of the elastic properties of a porous rock on the compressibility of the pore fluid: Geophysics, 40, 608-616.

Carroll, M. M., and N. Katsube, 1983, The role of Terzaghi effective stress in linearly elastic deformation: Journal of Energy Resources Technology, 105, 509-511.

Ciz, R., M. Urosevic, and K. Dodds, 2005, Pore pressure prediction based on seismic attributes response to overpressure: Australian Petroleum Production Exploration Association Journal, 45, 449-456.

Dodds, K., D. N. Dewhurst, A. F. Siggins, R. Ciz, M. Urosevic, B. Gurevich, and D. H. Sherlock, 2007, Experimental and theoretical rock physics research with application to reservoirs, seals and fluid processes: Journal of Petroleum Science and Engineering, 57, 16-36.

Dutta, N. C., 2002, Geopressure prediction using seismic data, Current status and the road ahead: Geophysics, 67, 2012-2041.

Eberhardt-Phillips, D., D.-H. Han, and M. D. Zoback, 1989, Empirical relationships among seismic velocity, effective pressure porosity, and clay content in sandstone: Geophysics, 54, 82-89.

Gassmann, F., 1951, Über die Elastizität poröser Medien: Vierteljahrsschrift der Naturforschende Gesellschaft, 96, 1-23.

Gurevich, B., 2004, A simple derivation of the effective stress coefficient for seismic velocities in porous rocks: Geophysics, 69, 393-397.

Hashin, Z., and S. Shtrikman, 1963, A variational approach to the elastic behavior of multiphase materials: Journal of the Mechanics and Physics of Solids, 11, 127-140.

Huffman, A. R., 2002, The future of pore-pressure prediction using geophysical methods: The Leading Edge, 21, 199-205.

Landau, L. D., and E. M. Lifshitz, 1959, Theory of elasticity: Pergamon Press, Inc.

Landrø, M., 2001, Discrimination between pressure and fluid saturation changes from timelapse seismic data: Geophysics, 66, 836-844.

Mavko, G., T. Mukerji, and J. Dvorkin, 1998, The rock physics handbook: Cambridge University Press.

Pilant, W. L., 1979, Elastic waves in the earth: Elsevier Science Publ. Co., Inc.

Prasad, M., and M. H. Manghnani, 1997, Effects of pore and differential pressure on compressional wave velocity and quality factor in Berea and Michigan sandstones: Geophysics, 62, 1163-1176.

Robin, P.-Y. F., 1973, Note on effective pressure: Journal of Geophysical Research, 78, 2434-2437.

Sayers, C. M., G. M. Johnson, and G. Denyer, 2002, Predrill pore-pressure prediction using seismic data: Geophysics, 67, 1286-1292.

Shapiro, S. A., 2003, Elastic piezosensitivity of porous and fractured rocks: Geophysics, 68, 482-486.

Siggins, A. F., and D. N. Dewhurst, 2003, Saturation, pore pressure and effective stress from sandstone acoustic properties: Geophysical Research Letters, 30, L1089.

Terzaghi, K., 1948, Theoretical soil mechanics: Wiley.

Todd, T., and G. Simmons, 1972, Effect of pore pressure on the velocity of compressional waves in low porosity rocks: Journal of Geophysical Research, 77, 3731-3743.

Tura, A., and D. Lumley, 1999, Estimating pressure and saturation changes from time-lapse AVO data: 69th Annual International Meeting, SEG, Expanded Abstracts, 1655-1658.

Vasco, D. W., 2004, Seismic imaging of reservoir flow properties, Timelapse pressure changes: Geophysics, 69, 511-521.

Wyllie, M. R. J., A. R. Gregory, and G. H. F. Gardner, 1958, An experimental investigation of factors affecting elastic wave velocities in porous media: Geophysics, 23, 459-493.

Zimmerman, R. W., 1991, Compressibility of sandstones: Elsevier Science Publ. Co., Inc. 